from an elderly patient with non-cirrhotic hepatitis $\mathrm{C}$ that needs further evaluations.

Methods A 78-year-old man was admitted due to right upper quadrant pain, mild fever and weight loss. Triple-phase abdominal CT showed a large liver mass $(12.8 \times 8.6 \times 10.2$ $\mathrm{cm}$ in size) with perihepatic fluids and multiple cystic lesions in various sizes that were conglomerated inside the mass (figure 1). Multiple satellites nodules with central cysts were also found, suggesting either hepatocellular carcinoma (HCC) with intrahepatic metastasis or multiple liver abscesses. He had elevated leucocytes $\left(15,570 \mathrm{~mm}^{-3}\right)$ and alanine transaminase $(244$ $\mathrm{U} / \mathrm{L}$ ). However, his serum seramoeba and fecal amoeba tests were negative. His Anti-HCV serology was positive. Moreover, his AFP and CEA levels were $75.94 \mathrm{ng} / \mathrm{mL}$ and $1.8 \mathrm{ng} / \mathrm{mL}$ consecutively. Two weeks course of antibiotics were initiated with no changes in his liver masses after further evaluation of abdominal CT. However, his leucocytes and alanine transaminase improved towards normal levels. Liver biopsy was offered for the patient, but he refused to do so.

Results The presentation of HCC can sometimes mimic an abscess, especially when cystic degeneration appeared within the tumor. Meanwhile, there were some cases of liver abscess that were indistinguishable from HCC. The patient, in this case, had fever and leucocytosis, which were the characteristics for liver abscess. Moreover, his AFP level was not increased significantly. However, insignificant changes after antibiotic therapy raised a dilemma on his definite diagnosis. Although very rare, a non-cirrhotic hepatocellular carcinoma in hepatitis $\mathrm{C}$ can also occur. This direct carcinogenesis mechanism can be potentially induced by the non-structural protein of hepatitis $\mathrm{C}$ virus. Looking at this case, a liver biopsy is needed for concluding a diagnosis for this patient.

Conclusions Establishing a diagnosis for atypical presentations of HCC and liver abscess can be quite difficult. Due to the big differences in their prognosis, familiarity with unusual clinical and imaging findings, as well as liver biopsy, are needed to ensure a certain diagnosis for the patient.

\section{IDDF2020-ABS-0189 CLINICAL ANALYSIS OF LAPS IN THE TREATMENT OF HEPATITIS B VIRUS- RELATED HEPATOCELLULAR CARCINOMA}

Zebin Chen*, Kai Lei, Shunli Shen, Baogang Peng. Department of Liver Surgery, the First Affiliated Hospital, Sun Yat-sen University, China

\subsection{6/gutjnl-2020-IDDF.164}

Background The aim of the study is to assess the efficacy and safety of laparoscopic microwave ablation and portal vein ligation for staged hepatectomy (LAPS) in the treatment of hepatitis B virus-related hepatocellular carcinoma.

Methods Clinical data of patients with hepatitis B virus-related liver cancer who underwent LAPS or ALPPS surgery in the First Affiliated Hospital of Sun Yat-sen University from April 2013 to September 2019 were retrospectively analyzed. In the study, all patients signed informed consents and were approved by local ethics committees. Statistical analysis of data were performed by the SPSS 26.0.

Results 7 patients with HCC underwent LAPS, while 14 patients underwent ALPPS surgery during the same period. $42.9 \%$ of patients had a single tumor, and the median tumor diameter was $96 \mathrm{~mm}$ (range: $26-151 \mathrm{~mm}$ ), the median preoperative FLR was 29.0\% (range19.5\%-38.8\%). The severe morbidity rates of patients undergoing LAPS was lower than those receiving ALPPS after the first operation $(P=0.009)$. The median time to first passage of flatus of patients in the ALPPS group and LAPS group was $3 \mathrm{~d}$ and $2 \mathrm{~d}(P=0.037)$ and the median time to out of bed was $4 \mathrm{~d}$ and $2 \mathrm{~d}(P=0.040)$. 6 patients in the LAPS group completed the second stage operation, and 11 patients in the ALPPS group completed the second stage operation $(P=0.694)$. The median interval was 15 days in the LAPS group and 11 days in the ALPPS group $(P=0.006)$. The incidence of severe complications was $36.4 \%$ in the ALPSS group and $50.0 \%$ in the LAPS group after the second operation $(P=0.615)$. One patient in the ALPPS group died after the second operation. There was no difference in FLR growth rate, operative time and intraoperative loss between the two groups.

Conclusions LAPS has potential advantages over classical ALPPS in reducing postoperative liver failure and severe complications. LAPS may have the same effect in promoting the growth of FLR in patients with hepatitis B virus-related HCC, with a longer interval between the two operations.

\section{IDDF2020-ABS-0192 TERLIPRESSIN NON-RESPONSE PREDICTS MORTALITY IN ACUTE-ON-CHRONIC LIVER FAILURE-A PROSPECTIVE COHORT STUDY}

Anand Kulkarni*, TR Sowmya, Mithun Sharma, Pramod Kumar, Samragni Vasireddy, Harshavardhan Tevethia, Nagaraja Rao Padaki, D Nageshwar Reddy. AlG Hospitals, Asian Institute of Gastroenterology, India

\subsection{6/gutjnl-2020-IDDF.165}

Background Terlipressin with albumin is the recommended standard of care for hepatorenal syndrome. However, there is a paucity of Asian data on the use of terlipressin in acute-onchronic liver failure (ACLF) patients. We aimed to evaluate the safety and efficacy of terlipressin infusion in ACLF patients with HRS-AKI (acute kidney injury).

Methods Consecutive ACLF patients aged between 18-75 years treated with terlipressin infusion for HRS-AKI were included. The primary objective was to assess the incidence of adverse events, and secondary were to assess the response to terlipressin therapy, predictors of terlipressin non-response, and transplant-free survival at day 30, 90.

Results During the study period, 116 ACLF patients (Males-

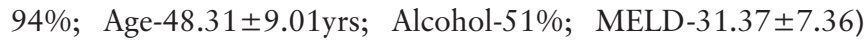
received terlipressin therapy for HRS-AKI. Fifty-two percent had a bacterial infection at baseline. The mean dose of terlipressin was $2.75 \pm 0.93 \mathrm{mg} /$ day for $5.28 \pm 3.51$ days. Twentyone percent of patients developed adverse effects (Diarrhea$37.5 \%$, abdominal pain-25\%) to terlipressin, and $12 \%$ required drug discontinuation. Sixty-five percent $(75 / 116)$ of patients responded to terlipressin, of which $39.7 \%(46 / 116)$ had a complete response, and 25\% (29/116) achieved a partial response. Time to the reversal of AKI was $4.8 \pm 2.64$ days. Twenty-eight percent of patients required renal replacement therapy. Transplant free survival at day 30 and 90 was $71 \%$ and $57.8 \%$. On multivariate analysis baseline serum creatinine [OR-2.24 (1.41-3.57);p<0.001], ACLF grade [Gr.II-4.98 (1.5$16.5) ; \mathrm{p}=0.009$, Gr.III-7.61 (1.91-30.16); $\mathrm{p}=0.004]$, and change in MAP at day 3 [OR-0.73 $(0.57-0.92) ; \mathrm{p}=0.009]$ were predictors of terlipressin non-response. On multivariate analysis terlipressin non-response [HR-3.49 (1.85-6.57); p<0.001] and 
$\mathrm{ROC}$ curve of $\mathrm{sCr}$ for predicting terlipressin non-response

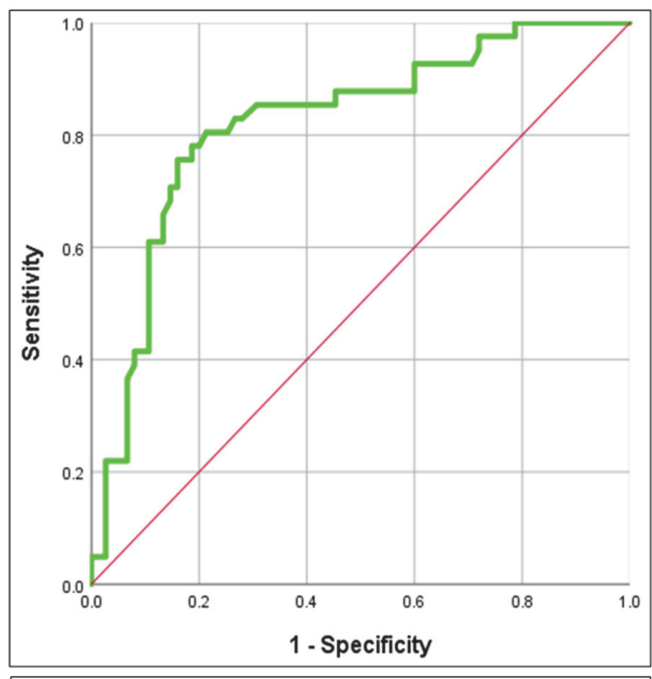

Baseline $\mathrm{sCr} \geq 3.02 \mathrm{mg} / \mathrm{dl}$ had a sensitivity of $75.6 \%$ and specificity of $84 \%$ for predicting terlipressin non-response with a AUROC of 82.2 $(95 \% \mathrm{CI}, 74.1-90.4 ; \mathrm{p}<0.001)$.
ROC curve of MELD score for predicting 90-day mortality

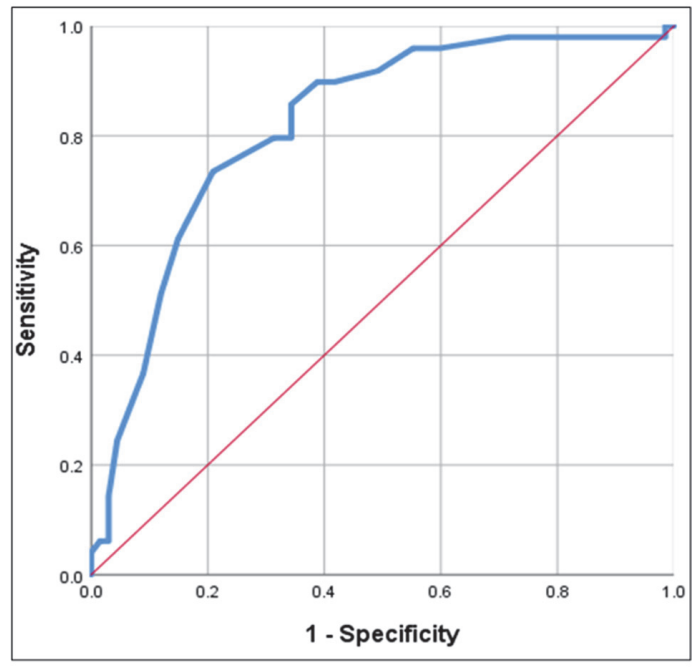

MELD $\geq 30.5$ had a sensitivity of $85.7 \%$ and specificity of $65.7 \%$ for predicting 90-day mortality with AUROC of 81.9 (95\%CI, 74.1-89.7; $\mathrm{p}<0.001)$.

Abstract IDDF2020-ABS-0192 Figure 1 ROC curve of serum creatinine (sCr) and MELD score for predicting terlipressin non-response and 90-day mortality, respectively

MELD score [HR-1.12 (1.06-1.18);p<0.001] predicted mortality at day-90 (figure 1).

Conclusions Terlipressin infusion is safe and effective in ACLF patients. Non-response to terlipressin is dependent on baseline $\mathrm{sCr}$ and ACLF grade. Terlipressin non-response and MELD scores predict mortality in ACLF patients with HRS-AKI. (CTRI/2019/10/021737).

\section{IDDF2020-ABS-0197 STUDY ON CORRELATION BETWEEN SERUM FERRITIN LEVELS AND LIVER STIFFNESS ASSESSED BY FIBROSCAN IN PATIENTS WITH CHRONIC HEPATITIS C}

${ }^{1} \mathrm{Ha}$ Phuong Linh*, ${ }^{2}$ Le Thi Thu Hien, ${ }^{1}$ Dinh Cong Dang, ${ }^{1}$ Hoang Van Tiep. 'Department of Internal Medicine, 103 Cam Khe Clinic, Vietnam; ${ }^{2}$ Department of Internal Medicine, Thai Nguyen University of Medicine and Pharmacy, Vietnam

\subsection{6/gutjnl-2020-IDDF.166}

Background Chronic hepatitis $\mathrm{C}$ is a major infectious disease which mainly causes of morbidity worldwide in patients with liver disease and liver transplantation. Raised ferritin levels play an important role in intervening the process which is associated with hepatic injury. Screening with non-invasive strategies can detect the disease at the early stage, and intervention could be initiated.

Aims To determine the correlation between serum ferritin levels and liver stiffness values in patients of chronic hepatitis C. Methods A cross-sectional study was conducted at 103 Cam Khe Clinic from May 2019 to April 2020. 93 patients with chronic hepatitis $\mathrm{C}$ fulfilling inclusion criteria were included in this study. Liver fibrosis stages were appreciated using transient hepatic elastography by Fibroscan, the activities of serum liver function biomarker enzymes and serum ferritin levels were determined by the automated analyser.

Results The average age of patients was 48 years, with men accounted for $78 \%$ of the total. The mean serum ferritin value was $148.19 \mathrm{ng} / \mathrm{ml}$, liver stiffness measurements range from 12.5 to $75.5 \mathrm{kPa}$, with a median value of $17.39 \pm$ $15.98 \mathrm{kPa}$. Significantly elevated levels of serum ferritin $(\mathrm{p}<$ 0.001), were detected in patients with severe fibrosis compared to mild fibrosis. The concentration of serum ferritin was increased with the evolution of fibrosis in all stages from F0 to $F 4$, and this increase was significant $(p<0.01)$ in cirrhotic patients (F4). There was a positive correlation between serum level of ferritin and the progression of fibrosis (0.979391) ( $\mathrm{r}=0.976)$.

Conclusions There is a significant correlation between serum ferritin and liver stiffness. Serum ferritin concentration may be used as liver fibrosis biomarkers.

\section{IDDF2020-ABS-0198 STUDY ON FIBROSIS CHANGE WITH TRANSIENT ELASTOGRAPHY IN CHRONIC HEPATITIS B VIRUS TREATMENT WITH TENOFOVIR}

${ }^{1}$ Ha Phuong Linh*, ${ }^{2}$ Le Thi Thu Hien, ${ }^{1}$ Hoang Van Tiep, ${ }^{2}$ Dinh Cong Dang, ${ }^{2}$ Nguyen Khac Hung Manh. 'Department of Internal Medicine, 103 Cam Khe Clinic, Vietnam: ${ }^{2}$ Department of Internal Medicine, Thai Nguyen University of Medicine and Pharmacy, Vietnam

\subsection{6/gutjpl-2020-IDDF.167}

Background Tenofovir disoproxil fumarate (TDF) is one of the first optimal choices to be used in the treatment of chronic hepatitis B. FibroScan is non-invasive methods to assess liver fibrosis.

Aims To evaluate the therapeutic effect of TDF on fibrosis via FibroScan after treatment.

Methods This study was conducted in 63 chronic hepatitis B patients who had the indication of antiviral therapy at 103 Cam Khe Clinic from March 2019 to March 2020. All patients with chronic hepatitis B treated with TDF for 6 months. Liver fibrosis stages were appreciated using transient hepatic elastography by Fibroscan before and after 6 months of treatment. 